\title{
Thermal conductivity enhancement of laser induced graphene foam upon P3HT infiltration
}

\author{
M. K. Smith, ${ }^{1}$ D. X. Luong, ${ }^{2,3}$ T. L. Bougher, ${ }^{4}$ K. Kalaitzidou, ${ }^{1,4}$ J. M. Tour, ${ }^{3,5,6}$ \\ and B. A. Cola ${ }^{1,4, a)}$ \\ ${ }^{1}$ Materials Science and Engineering, Georgia Institute of Technology, Atlanta, Georgia 30332, USA \\ ${ }^{2}$ Applied Physics Program, Rice University, Houston, Texas 77005, USA \\ ${ }^{3}$ Department of Chemistry, Rice University, Houston, Texas 77005, USA \\ ${ }^{4}$ George W. Woodruff School of Mechanical Engineering, Georgia Institute of Technology, Atlanta, \\ Georgia 30332, USA \\ ${ }^{5}$ NanoCarbon Center, Rice University, Houston, Texas 77005, USA \\ ${ }^{6}$ Department of Materials Science and NanoEngineering, Rice University, Houston, Texas 77005, USA
}

(Received 8 September 2016; accepted 8 December 2016; published online 21 December 2016)

\begin{abstract}
Significant research has been dedicated to the exploration of high thermal conductivity polymer composite materials with conductive filler particles for use in heat transfer applications. However, poor particle dispersibility and interfacial phonon scattering have limited the effective composite thermal conductivity. Three-dimensional foams with high ligament thermal conductivity offer a potential solution to the two aforementioned problems but are traditionally fabricated through expensive and/or complex manufacturing methods. Here, laser induced graphene foams, fabricated through a simple and cost effective laser ablation method, are infiltrated with poly(3-hexylthiophene) in a step-wise fashion to demonstrate the impact of polymer on the thermal conductivity of the composite system. Surprisingly, the addition of polymer results in a drastic (250\%) improvement in material thermal conductivity, enhancing the graphene foam's thermal conductivity from $0.68 \mathrm{~W} / \mathrm{m}-\mathrm{K}$ to $1.72 \mathrm{~W} / \mathrm{m}-\mathrm{K}$ for the fully infiltrated composite material. Graphene foam density measurements and theoretical models are utilized to estimate the effective ribbon thermal conductivity as a function of polymer filling. Here, it is proposed that the polymer solution acts as a binding material, which draws graphene ligaments together through elastocapillary coalescence and bonds these ligaments upon drying, resulting in greatly reduced contact resistance within the foam and an effective thermal conductivity improvement greater than what would be expected from the addition of polymer alone. Published by AIP Publishing. [http://dx.doi.org/10.1063/1.4972790]
\end{abstract}

Thermal management of electronic devices is a burgeoning challenge due to their increased power consumption and reduced weight and size requirements, which ultimately result in high power density. In most electronic packages, there exist interfaces where heat generating components must be connected to heat sinking materials using thermal interface materials (TIMs). TIMs enhance the thermal conductance from one component to the other by filling in air gaps (caused by microscopic surface roughness and tolerance mismatch) that would otherwise form thermally insulating air pockets, and most commercial TIMs are composed of a polymer matrix infiltrated with a high conductivity filler. While the specific application greatly influences the desired material properties, it is generally ideal for thermal interface materials to be mechanically compliant (soft) and have high thermal conductivity. "Softness" (or the ability to deform under pressure) allows TIMs to fill in surface roughness crevices and improve the effective heat transfer between substrates; however, soft materials with high thermal conductivity are difficult to manufacture due to the low intrinsic thermal conductivity of polymers, typically between 0.1 and $0.5 \mathrm{~W} / \mathrm{m}-\mathrm{K}^{2}$ (with the exception of recent efforts to increase the thermal conductivity of pure polymer, where up to $1.5 \mathrm{~W} / \mathrm{m}-\mathrm{K}$ has been achieved using engineered interchain,

${ }^{\text {a)} E l e c t r o n i c ~ m a i l: ~ c o l a @ g a t e c h . e d u ~}$ heat conducting bonds ${ }^{3}$ ). To overcome this challenge, commercial TIM manufacturers and academic researchers generally fill the polymer matrix with thermally conductive particles such as boron nitride or aluminum oxide particles (for electrically insulating applications) ${ }^{4,5}$ or various forms of carbon such as carbon fibers, ${ }^{6}$ carbon nanotubes, ${ }^{7}$ and graphene flakes ${ }^{8}$ and metals including $\mathrm{Ag}^{9}$ (for applications where electrical isolation is less critical). Although the aforementioned filler particles exhibit large thermal conductivities, the effective increase in composite thermal conductivity can be low because of poor particle dispersion and contact resistance, which includes both phonon scattering at the polymer matrix and filler particle interface and voiding or incomplete wetting of the particle surface. ${ }^{10}$ To achieve significantly enhanced through plane (z-axis) thermal conductivity, the resultant composite material requires high filler particle concentration, to the point where filling begins to compromise mechanical properties, processability, and greatly increases material cost. For example, even at filler particle volume fractions above $50 \mathrm{vol} \%$, the effective through plane thermal conductivity is still often an order of magnitude or more below the thermal conductivity of the filler particles. ${ }^{11,12}$

Porous graphite and graphene foams (GF) with a threedimensionally connected network of graphene (or graphite) ligaments have been investigated to provide high through 
plane thermal conduction as free standing foams or when combined with secondary polymer to form a composite. ${ }^{13,14}$ The percolated skeletal carbon ligaments provide heat transport pathways with no interfacial contact points and hence reduced interfacial phonon scattering, and graphene foam composite systems have demonstrated ligament thermal conductivities greater than $1600 \mathrm{~W} / \mathrm{m}-\mathrm{K}^{15}$ In addition, free standing graphene foams, with ligament thermal conductivity estimated to be $500 \mathrm{~W} / \mathrm{m}-\mathrm{K}$, have demonstrated high effective thermal conductivity under compression and exceptional performance in thermal interface applications. ${ }^{16}$ However, expensive, high temperature and time consuming CVD or pyrolytic methods are often used to fabricated graphene foams, pricing graphene foam based TIMs out of the commercial market ${ }^{13-16}$ (high performance TIMs typically sell for between $\$ 0.5$ and $\$ 5.00 /$ in. $^{2}$ whereas graphene foams are currently sold for greater than $\$ 50 /$ in. $^{2}$ (Graphene Supermarket)). Here, we investigate thermal transport in low cost laser induced graphene (LIG) foam and LIG/poly(3-hexylthiophene-2,5-diyl) (P3HT) composites fabricated through laser ablation and drop casting methods. The polymer P3HT has high temperature stability (melting point above $200^{\circ} \mathrm{C}$ ) and strong pi-pi interactions with graphene structures, which allows for good foam wetting and infiltration. ${ }^{17}$ The thermal conductivity of bare LIG foam is measured as a function of laser power and then thin coatings of P3HT are incrementally added to the LIG foam network and an unusually high increase in thermal transport is observed. These interesting results serve to the further conversation of graphene foams and composites for thermal management applications.

LIG foams are produced using a simple laser fabrication technique described previously. ${ }^{18}$ In short, the foams are manufactured from commercially available $130 \mu \mathrm{m}$ thick Kapton polyimide (PI) sheets using a $\mathrm{CO}_{2}$ infrared laser where laser irradiation results in the photothermal conversion of $\mathrm{sp}^{3}$-carbon atoms to $\mathrm{sp}^{2}$-carbon atoms and the formation of a three-dimensional graphene network on the surface of the PI film. The $\mathrm{CO}_{2}$ laser power is altered to control the degree of graphitization and resultant graphene foam thickness and porosity. ${ }^{18}$ The entire process is performed under ambient conditions for the conversion of PI films into porous graphene foam where the porosity originates from the outgassing during the high temperature laser process. The LIG process is unique to a small class of polymers, particularly those that bear the aromatic and imide repeat units due to an overlap of the $10.6 \mu \mathrm{m} \mathrm{CO} \mathrm{CO}_{2}$ laser line with an absorbance shoulder in the PI. For this study, the laser duty cycle is adjusted from $4 \%$ to $10 \%$ in $2 \%$ increments using a $60 \mathrm{~W}$ peak laser, operating in pulse width modulation (PMW) at $6 \mathrm{kHz}$ and a laser spot size of $100 \mu \mathrm{m}$. As laser power density increases, the LIG thickness on the surface of PI film increases and the porosity increases. The resultant LIG thermal conductivity is measured as a function of laser power using the photoacoustic (PA) method. The PA method is a photothermal measurement procedure where a modulated laser strikes the surface of a material housed in a pressurized chamber and as the sample surface heats, it conducts heat into the gas near its surface and the gas expands sending acoustic waves throughout the chamber. ${ }^{19}$ The phase and amplitude of the acoustic signal are then compared to a reference material, and a one-dimensional heat conduction model is used to determine the materials' thermal properties. ${ }^{20}$ Further information regarding the PA measurement technique, model fitting parameters, and sample preparation can be found in the supplementary material and our previous publications. $^{21-23}$

As illustrated in Figure 1, the thermal conductivity does not significantly change as a function of laser irradiation, and a peak value of approximately $0.7 \mathrm{~W} / \mathrm{m}-\mathrm{K}$ is observed for foams fabricated at $4 \%$ duty cycle. Regardless, the LIG foam thermal conductivity is significantly larger than the PI thermal conductivity of $0.15 \mathrm{~W} / \mathrm{m}-\mathrm{K}$ reported by DuPont. The foam thickness increases gradually from a minimum of approximately $27 \mu \mathrm{m}$ to a maximum of approximately $50 \mu \mathrm{m}$ as the laser duty cycle increases from $4 \%$ to $10 \%$. Scanning electron micrographs of the LIG surface as a function of laser power (supplementary material) demonstrate that the characteristic surface porosity dimensions (the size of surface voids) are well below the PA laser spot size of $1 \mathrm{~mm}$ in diameter ( $>97 \%$ of surface area of $6 \%$ sample exhibits pore size $<9 \mathrm{~nm}$ (Ref. 18)), which is important for determining the applicability of this thermal testing method.

Although all thermal measurements fall within the same uncertainty bounds, the trend suggests that maximum foam thermal conductivity is achieved at a laser duty cycle of $6 \%$, most probably due to competing material property modifications that occur as the laser power is increased. It is expected (and has been confirmed by (Lin et al.)) that at low laser power the foam will have the highest density and lowest porosity, but lower relative levels of graphitization; whereas at high laser power ( $8 \%-10 \%$ duty cycle) the opposite is expected (high levels of graphitization, but lower graphene ligament density and high porosity). ${ }^{18}$ This expectation is illustrated when comparing points for the $4 \%$ and $8 \%$ duty cycles in Figure 1, where nearly identical thermal conductivity is achieved despite expected variance in density. The results of the PA measurements suggest that the 6\% laser power provides an ideal medium where both graphitization and density are relatively high, resulting in peak LIG thermal conductivity. Hence, the $6 \%$ LIG is expected to produce the

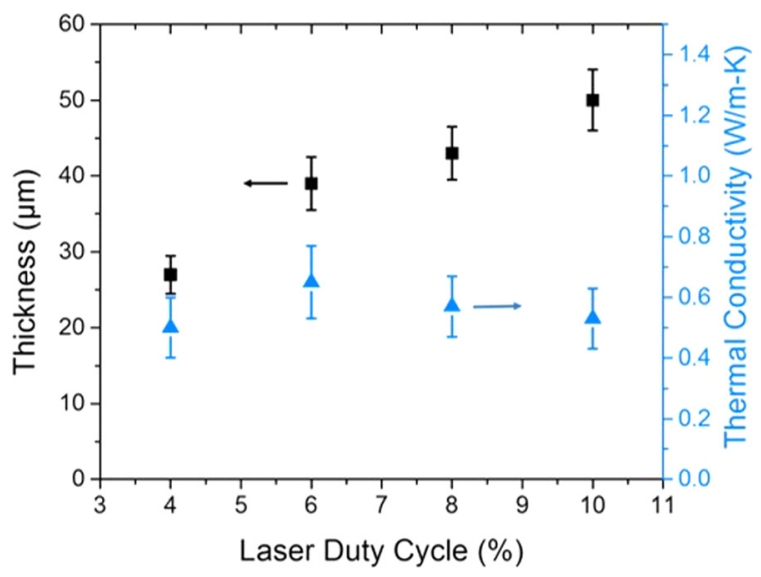

FIG. 1. Graphene foam thickness (black squares) and thermal conductivity (blue triangles) as a function of laser power. The thickness error bars represent the standard deviation of three cross-sectional SEM images and the thermal conductivity error bars represent the combined measurement and fitting uncertainty (see Ref. 21 for more details). 
highest thermal conductivity when combined with polymer and was chosen as the base material to fabricate LIG/P3HT composites for thermal testing.

Regioregular P3HT (purchased from Sigma, product number 445703) is stirred into chlorobenzene (using a hot plate set at $60^{\circ} \mathrm{C}$ and for $1 \mathrm{~h}$ of stirring) to form a $15 \mathrm{mg} / \mathrm{ml}$ P3HT solution. An adjustable volume micropipette is used to apply P3HT to the surface of $6 \%$ duty cycle GF in $75 \mu \mathrm{l}$ increments and dried for $2 \mathrm{~h}$ in the ambient environment. The resulting P3HT vol. \% is calculated using the measured volume of the graphene foam and the known amount of P3HT added. The LIG becomes saturated with polymer and a P3HT film begins to develop on the sample surface at the highest P3HT vol. \% filling, as illustrated in the inset images in Figure 2. However, cross-sectional SEM images reveal the presence of voids that exist even after full sample saturation, resulting in a lower than expected peak P3HT filling of approximately $55 \mathrm{vol} . \%$ of the total. Hence, the authors believe that the proposed method results in maximum achievable infiltration, as SEM imaging indicates the presence of P3HT films at the top, bottom, and through the entire sample thickness and the addition of more polymer will only result in further surface accumulation. The remaining voids are likely due to sections of the graphene foam that are impenetrable to polymer infiltration due to fully enclosed wall structures or substantially small feature sizes. Figures 2 shows that the GF thermal conductivity improves from approximately $0.7 \mathrm{~W} / \mathrm{m}-\mathrm{K}$ at no P3HT filling to an unexpectedly high value of approximately $1.7 \mathrm{~W} / \mathrm{m}-\mathrm{K}$ with maximum achievable infiltration.

The dramatic increase in thermal conductivity is surprising considering that the thermal conductivity of P3HT is approximately $0.28 \mathrm{~W} / \mathrm{m}-\mathrm{K}$ (measured by PA on dropcast films) and the thermal conductivity of the helium gas in the PA testing chamber is $0.15 \mathrm{~W} / \mathrm{m}-\mathrm{K} .{ }^{24}$ Hence, replacing the helium gas within the GF matrix with P3HT should not result

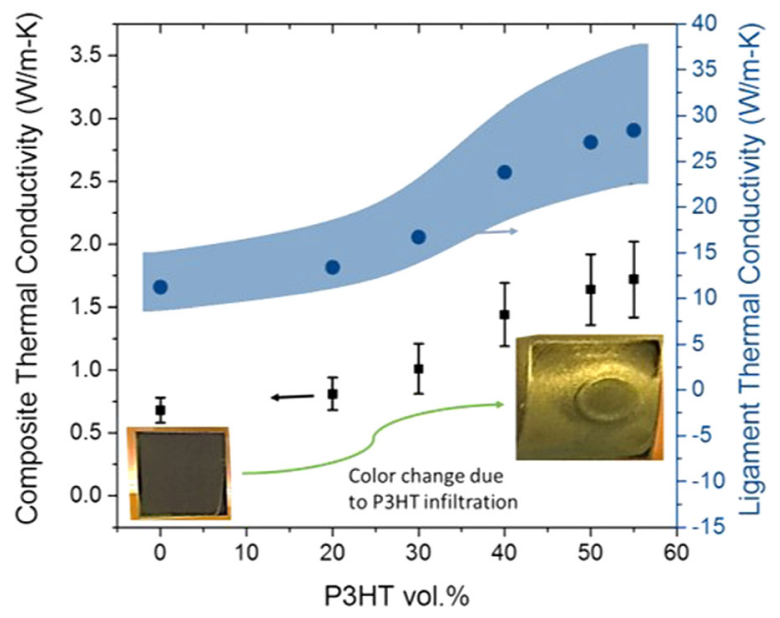

FIG. 2. Thermal conductivity of the graphene foam/P3HT composite as a function of P3HT vol. \% is illustrated by the black squares corresponding to the left vertical axis. The error bars represent the uncertainty associated with the measurement and data fitting uncertainty. The blue shaded region corresponds to the right vertical axis and represents the theoretically calculated ligament thermal conductivity with upper and lower bounds represented by the uncertainty in LIG foam density measurements. The inset images display the LIG surface before and after maximum P3HT infiltration. in a significant increase in composite thermal conductivity above the $\sim 0.7 \mathrm{~W} / \mathrm{m}-\mathrm{K}$ value observed at no P3HT infiltration. A high precision scale is used to measure the weight of $1 \mathrm{~cm}^{2}$ sections of LIG on PI, and a scanning electron microscope is used to measure the thickness of each respective layer. These measured values and the manufacturer specified density of the PI film $\left(1.42 \mathrm{~g} / \mathrm{cm}^{3}\right)$ are then used to calculate the LIG density. Using the measured $6 \%$ duty cycle LIG density and the measured bulk thermal conductivity as a function of polymer filling $\left(\lambda_{\text {Bulk }}\right)$, the estimated ligament thermal conductivity $\left(\lambda_{\text {Solid }}\right)$ is calculated and plotted in Figure 2 utilizing a simple analytical model to predict the thermal conductivity of connected graphite networks ${ }^{15}$ (Equation (1))

$$
\lambda_{\text {Bulk }}=\alpha\left(\frac{\rho_{\text {Bulk }}}{\rho_{\text {Solid }}}\right)^{M} \lambda_{\text {Solid }} .
$$

In Equation (1), $\alpha$ (the cellular structure coefficient, equal to 0.734 ) and $M$ (equal to 1.427) are constants to account for the impact of both the pore shape and the volume ratio of ligaments to junctions on the path length of heat flow in GF, respectively. These constants were derived for CVD grown graphitic foam networks and may not exactly correlate with our material system; however, because our material has a relatively high density and low bulk thermal conductivity, simpler models (Equations (1) and (2) in Ref. 15) with constants that are universally applied to all foam systems produce results within the uncertainty bounds associated with our density measurements. The bulk density (GF density) and the solid density (graphene ligament density) are both denoted by $\rho$ where the bulk is measured to be $385 \pm 70 \mathrm{mg} / \mathrm{cm}^{3}$ and the ligament value is taken from the literature as $2.2 \mathrm{~g} / \mathrm{cm}^{3}{ }^{15}$ The blue shaded region represents the upper and lower bounds of the estimated ligament conductivity as a function of GF density, where the bounds are determined by the uncertainty in the density measurements.

The relatively low values of GF ligament thermal conductivity are likely due to the ultra-polycrystalline nature of the LIG foams and poor structural integrity (fractured ligaments), which will both contribute to enhanced interfacial phonon scattering and contact resistance. The dramatic increase in thermal conductivity with addition of P3HT is likely due to a significant enhancement of the graphene foam ligament network thermal conductivity, where effective ligament thermal conductivity increases from approximately $12 \mathrm{~W} / \mathrm{m}-\mathrm{K}$ to $28 \mathrm{~W} / \mathrm{m}-\mathrm{K}$. We hypothesize that the addition of polymer solution results in capillary driven healing of broken ligament networks through elastocapillary coalescence ${ }^{25,26}$ allowing for an increase in the total number of thermal transport pathways and better adhesion between contact points of pathways in physical contact. Electrocapillary coalescence occurs when solvent induced capillary forces overcome LIG network elastic forces and draw LIG ligaments into contact (or coalescence) and then the surfaces remain in contact after removal of solvent due to adhesion or polymer binding forces. Multiple wetting events were recorded with solvent only and the resulting GF thermal conductivity did not change significantly, which suggest that the polymer is essential due to its strong interaction with the graphene and 
its ability to serve as a binding material upon solvent evaporation.

The theoretically calculated ligament thermal conductivity values $\left(\lambda_{\text {Solid }}\right)$, as well as the polymer thermal conductivity $\left(\lambda_{\text {Polymer }}\right)$, is used in a normalized unit cube model developed by Yu et al..$^{27}$ (Equation (2)) to calculate the bulk composite thermal conductivity $\left(\lambda_{B u l k}\right)$

$$
\begin{aligned}
\lambda_{\text {Bulk }}= & \left(1-2 n+2 n^{2}\right)\left(\frac{(1 / n-1)^{2}+\sigma}{(1 / n-1)^{2}+1}\right) \lambda_{\text {Polymer }} \\
& +\frac{2 n(1-n)}{(1-n) \sigma+n} \lambda_{\text {Solid }},
\end{aligned}
$$

where $\sigma$ is the ratio of solid ligament thermal conductivity to the polymer thermal conductivity (or fluid thermal conductivity (helium gas) is the case of no polymer infiltration) and $n=2 \mathrm{c} / \mathrm{H}$ where $\mathrm{c}$ and $\mathrm{H}$ are the structural parameters determined by the foam porosity and pore diameter (further details are included in the original LIG fabrication publication's (Lin et al.) supplementary material ${ }^{18}$ ). The theoretically calculated composite thermal conductivities are illustrated by the green shaded region in Figure 3 and the measured LIG/P3HT composite thermal conductivities are indicated by the black squares and are plotted again (these are the same values from Figure 2) for comparison.

In general, good agreement is observed between the theoretically predicted values (calculated using the estimated LIG ligament thermal conductivities) and experimental photoacoustic measured values. However, at low filling volumes the model over-predicts the measured thermal conductivity whereas at the higher filling volumes the best agreement between theoretical and measured values is achieved. Less consistent agreement between the experimental and model results at low conductivities likely arises because the theoretically models were originally derived for foams with high ligament thermal conductivity $(>500 \mathrm{~W} / \mathrm{m}-\mathrm{K})$. Regardless, these results support the previous finding that the effective

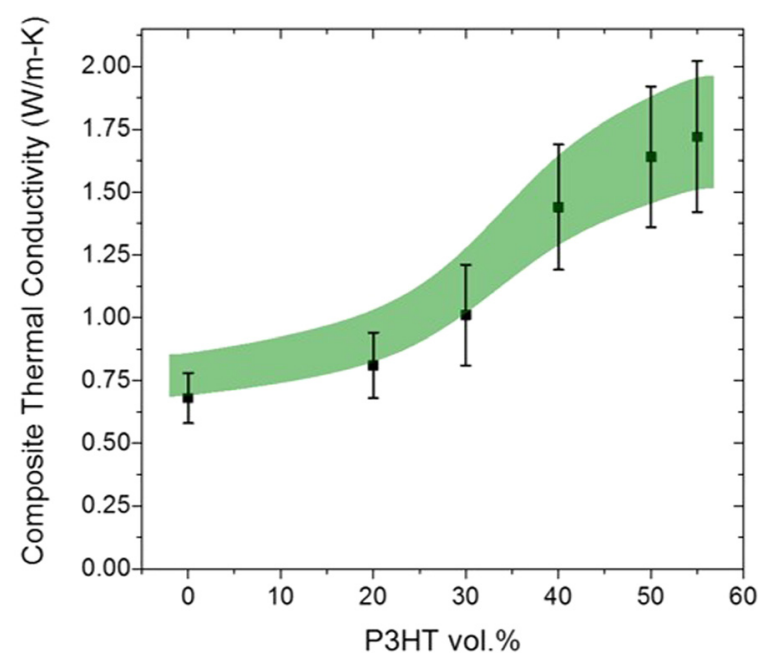

FIG. 3. Experimental (black squares) and theoretical (green shaded region) LIG thermal conductivity as a function of P3HT filling vol. \%. The error bars represent the combine measurement and fitting uncertainty associated with the PA method and the bounds on the shaded region are determine by the uncertainty in the LIG density measurements. ligament network thermal conductivity is improved at high P3HT vol. \% filling, resulting in improved composite thermal conductivity. Similar results were recently reported for graphene foam thermal conductivity as a function of temperature, where it was found that the GF thermal conductivity significantly increased with temperature due to a thermal expansion induced "tightening" of the foam network, which reduced contact resistance between ligaments. ${ }^{28}$ Here, we argue that elastocapillary coalescence during solution drying draws LIG ligaments into contact and the P3HT acts as a binding material to secure these contacts after full solvent evaporation, which results in a reduction in the effective skeletal thermal resistance, or an increase in the effective thermal conductivity. Three-dimensional graphene foam fabricated through CVD methods on sacrificial nickel support has demonstrated ligament thermal conductivity as high as $995 \mathrm{~W} / \mathrm{m}-\mathrm{K}$ and a foam conductivity of $1.70 \mathrm{~W} / \mathrm{m}-\mathrm{K}$ at a very low vol. \% of 0.45 (or more than an order of magnitude lower density $\left(11.6 \mathrm{mg} / \mathrm{cm}^{3}\right)$ as compared to LIG). ${ }^{29}$ Although CVD methods are expected to be more costly, they demonstrate the high potential for graphene foams in thermal conduction applications and the potential room for improvement in cheaper LIG foams, assuming that the LIG quality can be improved. In another study, graphene nanoplatelets with high quality and low defect density (manufacturer specified thermal conductivity of $3000 \mathrm{~W} / \mathrm{m}-\mathrm{K}$ ) were mixed into polymer matrix under high compression, resulting in composites with record high $12.4 \mathrm{~W} / \mathrm{m}-\mathrm{K}$ thermal conductivity at $25 \mathrm{vol} . \% .^{30}$ The observed $12.4 \mathrm{~W} / \mathrm{m}-\mathrm{K}$ value is impressive, but it is clear that the CVD graphene foam sample would achieve higher conductivity at an equivalent vol. \%, likely due to high thermal contact resistances in the randomly dispersed system. Hence, the LIG fabrication offers the potential to fabricate graphene foam with high vol. \% and may also avoid the interfacial contact resistance problem associated with randomly dispersed systems.

The results presented here demonstrate a simple method to drastically improve thermal transport in LIG foam composites. Moreover, the remarkable thermal conductivity enhancement from approximately $0.7 \mathrm{~W} / \mathrm{m}-\mathrm{K}$ to greater than $1.7 \mathrm{~W} / \mathrm{m}-\mathrm{K}$ suggests that this process could be interesting for commercial thermal interface applications, as well as electrical applications not discussed in this report. If the LIG manufacturing process can be optimized to attain higher quality graphene ligaments, the effective thermal conductivity of LIG/polymer composites may achieve significantly enhanced thermal conductivities to the point of viability in certain commercial thermal applications.

See supplementary material for further information regarding the PA measurement technique, model fitting parameters, and sample characterization.

This work was supported by the Georgia Tech TI:GER (Technology Innovation Generation Economic Results) Fellowship, the NSF sponsored Georgia Tech NESAC (Nanomaterials foe Energy Storage and Conversion) IGERT Traineeship, and by Samsung through a Samsung GRO grant. J.M.T. thanks the AFOSR for financial support (Nos. FA9550-14-1-0111 and FA9550-12-1-0035). 
${ }^{1}$ D. Chung, J. Mater. Eng. Perform. 10, 56 (2001).

${ }^{2}$ C. L. Choy, Polymer 18, 984 (1977).

${ }^{3}$ G.-H. Kim, D. Lee, A. Shanker, L. Shao, M. S. Kwon, D. Gidley, J. Kim, and K. P. Pipe, Nat. Mater. 14, 295 (2015).

${ }^{4}$ K. Yung and H. Liem, J. Appl. Polym. Sci. 106, 3587 (2007).

${ }^{5}$ C. P. Wong and R. S. Bollampally, J. Appl. Polym. Sci. 74, 3396 (1999).

${ }^{6}$ K. Uetani, S. Ata, S. Tomonoh, T. Yamada, M. Yumura, and K. Hata, Adv. Mater. 26, 5857 (2014).

${ }^{7}$ B. A. Cola, J. Xu, C. Cheng, X. Xu, T. S. Fisher, and H. Hu, J. Appl. Phys. 101, 054313 (2007)

${ }^{8}$ K. M. Shahil and A. A. Balandin, Nano Lett. 12, 861 (2012).

${ }^{9}$ X. Huang, P. Jiang, and L. Xie, Appl. Phys. Lett. 95, 242901 (2009).

${ }^{10}$ H. Chen, V. V. Ginzburg, J. Yang, Y. Yang, W. Liu, Y. Huang, L. Du, and B. Chen, Prog. Polym. Sci. 59, 41 (2016)

${ }^{11}$ J.-P. Hong, S.-W. Yoon, T. Hwang, J.-S. Oh, S.-C. Hong, Y. Lee, and J.-D. Nam, Thermochim. Acta 537, 70 (2012).

${ }^{12}$ N. Burger, A. Laachachi, M. Ferriol, M. Lutz, V. Toniazzo, and D. Ruch, Prog. Polym. Sci 61, 1 (2016).

${ }^{13}$ Z. Liu, D. Shen, J. Yu, W. Dai, C. Li, S. Du, N. Jiang, H. Li, and C.-T. Lin, RSC Adv. 6, 22364 (2016).

${ }^{14}$ J.-1. Song, Q.-g. Guo, Y.-j. Zhong, X.-q. Gao, Z.-h. Feng, Z. Fan, J.-1. Shi, and L. Liu, New Carbon Mater. 27, 27 (2012).

${ }^{15}$ J. W. Klett, A. D. McMillan, N. C. Gallego, and C. A. Walls, J. Mater. Sci. 39, 3659 (2004)

${ }^{16}$ X. Zhang, K. K. Yeung, Z. Gao, J. Li, H. Sun, H. Xu, K. Zhang, M. Zhang, Z. Chen, and M. M. Yuen, Carbon 66, 201 (2014).

${ }^{17}$ J. H. Taphouse, T. L. Bougher, V. Singh, P. P. S. S. Abadi, S. Graham, and B. A. Cola, Nanotechnology 24, 105401 (2013).
${ }^{18}$ J. Lin, Z. Peng, Y. Liu, F. Ruiz-Zepeda, R. Ye, E. L. G. Samuel, M. J. Yacaman, B. I. Yakobson, and J. M. Tour, Nat. Commun. 5, 5714 (2014).

${ }^{19}$ X. Wang, B. A. Cola, T. L. Bougher, S. L. Hodson, T. S. Fisher, and X. $\mathrm{Xu}$, "Photoacoustic technique for thermal conductivity and thermal interface measurements," in Annual Review of Heat Transfer (Begell House, Inc., Danbury, Connecticut, 2013), p. 135.

${ }^{20}$ H. Hu, X. Wang, and X. Xu, J. Appl. Phys. 86, 3593 (1999).

${ }^{21}$ M. K. Smith, V. Singh, K. Kalaitzidou, and B. A. Cola, ACS Appl. Mater. Interfaces 8, 14788 (2016).

${ }^{22}$ M. K. Smith, V. Singh, K. Kalaitzidou, and B. A. Cola, ACS Nano 9, 1080 (2015).

${ }^{23}$ V. Singh, T. L. Bougher, A. Weathers, Y. Cai, K. Bi, M. T. Pettes, S. A. McMenamin, W. Lv, D. P. Resler, T. R. Gattuso, D. H. Altman, K. H. Sandhage, L. Shi, A. Henry, and B. A. Cola, Nat. Nanotechnol. 9, 384 (2014).

${ }^{24}$ N. B. Vargaftik, Handbook of Thermal Conductivity of Liquids and Gases (CRC press, 1993).

${ }^{25}$ J. Bico, B. Roman, L. Moulin, and A. Boudaoud, Nature 432, 690 (2004).

${ }^{26}$ N. Chakrapani, B. Wei, A. Carrillo, P. M. Ajayan, and R. S. Kane, Proc. Natl. Acad. Sci. 101, 4009 (2004).

${ }^{27}$ Q. Yu, B. E. Thompson, and A. G. Straatman, J. Heat Transfer 128, 352 (2005).

${ }^{28}$ M. Li, Y. Sun, H. Xiao, X. Hu, and Y. Yue, Nanotechnology 26, 105703 (2015).

${ }^{29}$ M. T. Pettes, H. Ji, R. S. Ruoff, and L. Shi, Nano Lett. 12, 2959 (2012).

${ }^{30}$ M. Shtein, R. Nadiv, M. Buzaglo, K. Kahil, and O. Regev, Chem. Mater. 27, 2100 (2015) 